\title{
Impact of Optimum Diabetes Care on Safety of Fasting Ramadan in Patients with Gestational Diabetes
}

Mohamed Hassanein, Sona Abuelkheir, Manal Twair, Alaaeldin Bashier, Fauzia Rashid, Maryam Al Saeed, Fatima Alsayyah, Fawzi Altayb, Mohammed Abdellatif, Elamin Abdelgadir, Azza Khalifa, Fatheya Alawadi

\section{Background:}

Fasting during Ramadan is obligatory for all healthy adult Muslims. Specific groups of people are exempt from fasting, including pregnant women. However, many women with GDM insist on fasting despite advice against it as they are considered high-risk according to all guidelines.

The use of any type of continuous glucose monitoring (CGM) was not previously evaluated during Ramadan for GDM.

\section{Aim:}

The primary objective is to study the safety of fasting in patients with GDM in Ramadan in terms of glycemic fluctuations and changes in biometric and biochemical parameters before and after Ramadan.

\section{Methods:}

25 women with GDM insisting on fasting received optimum care for their diabetes control during Ramadan as follows:

- CGM monitoring with Freestyle Libre covering a duration of 6 weeks to include time before, during and after Ramadan.

- Ramadan and diabetes focused education session.

- Treatment was adjusted as per guidelines.

- Attendance of diabetes antenatal clinics every 1-2 weeks.

- Biometric and biochemical data were measured 2-4 weeks before and after Ramadan.

\section{Results and Discussion:}

$72 \%$ were Emirati; mean gestational age was 26.48 weeks. $66 \%$ fasted $21-30$ days, $32 \%$ were treated with diet only, $48 \%$ with metformin only and $20 \%$ with insulin and metformin.

Data comparing pre to post-Ramadan showed that mean weight increased by $1 \mathrm{~kg}$ while there was no significant change in blood pressure or e-GFR. Lipids parameters showed some increase where total cholesterol increased from 133.2 to $151.4 \mathrm{mg} / \mathrm{dl}$. Mean fructosamine was unchanged while HbA1c showed improvement from $6 \%$ to $5.5 \%$.

Average number of hypoglycemic events during Ramadan showed increment from 5 to 9 when compared to non-Ramadan. The duration between 12-18 hours showed the highest incidence of hypoglycemic events.

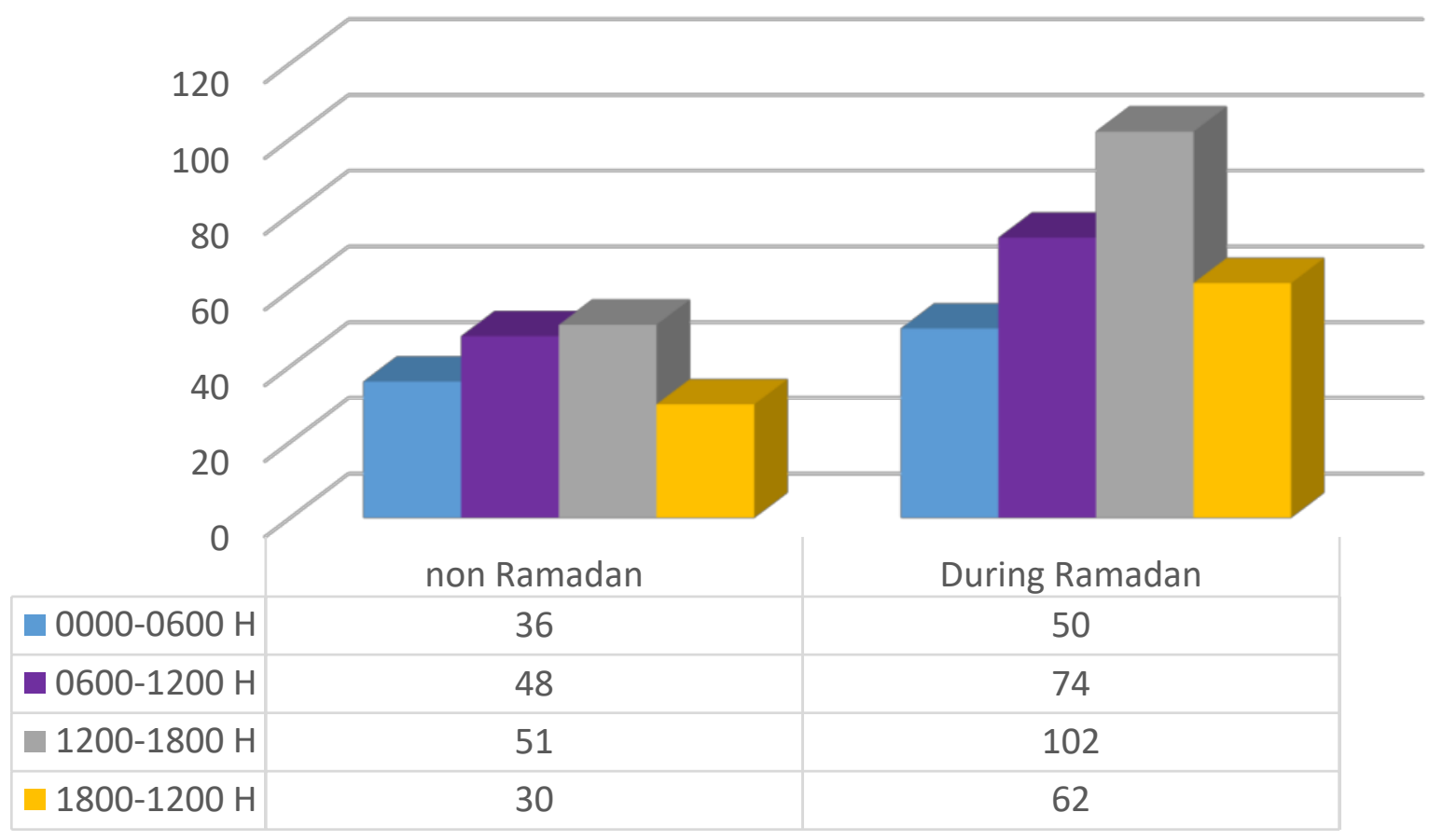

Total number of hypoglycemic events by time of day

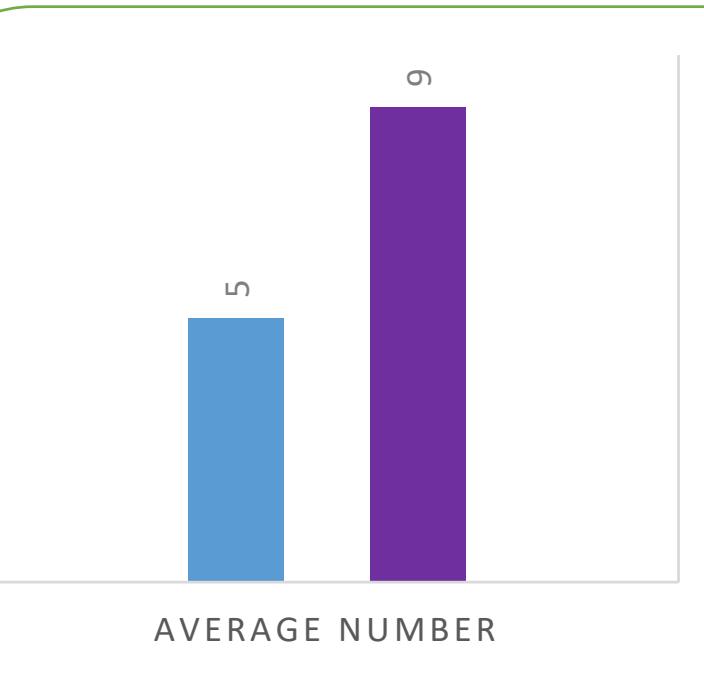

- Non Ramadan During Ramadan

Average number of hypoglycemic events in Ramadan vs Non Ramadan symptomatic - asymptomatic

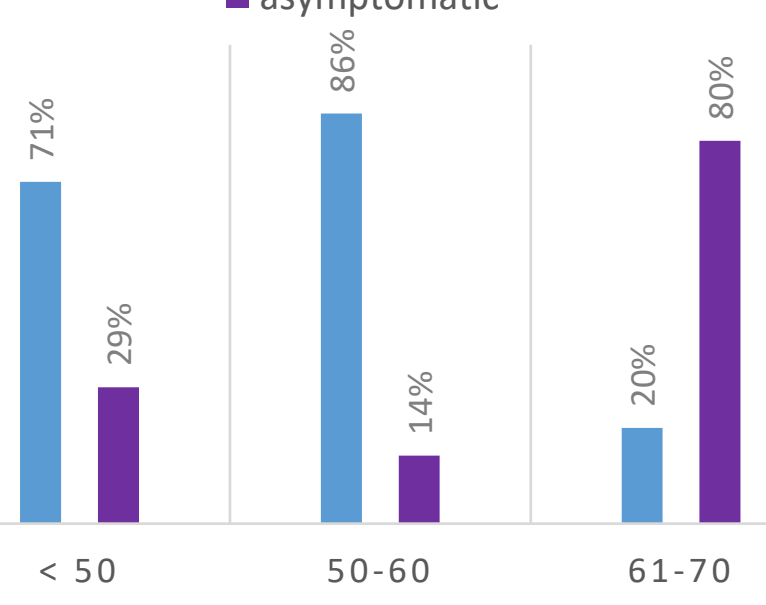

Symptomatic vs Asymptomatic Hypoglycemia During Ramadan

Those treated with diet only had 85 hypoglycemic events in Ramadan and 30 events in non-Ramadan period. Those on metformin only had 140 events in Ramadan versus 47 events in non-Ramadan, while those treated with insulin and metformin had 24 events in Ramadan vs 16 events in nonRamadan. Few patients were using SMBG at the same time as Freestyle Libre. In those who did, hypoglycemia was confirmed in 3 patients on diet, 7 patients on metformin and 2 for those on insulin with metformin.

\begin{tabular}{|lccl|}
\hline Type of Treatment & Diet & Metformin & $\begin{array}{l}\text { Metformin } \\
+ \text { insulin }\end{array}$ \\
\hline $\begin{array}{l}\text { \# hypoglycemic event } \\
\text { Ramadan }\end{array}$ & 85 & 140 & 24 \\
\hline $\begin{array}{l}\text { \# hypoglycemic event } \\
\text { non Ramadan }\end{array}$ & 30 & 47 & 16 \\
\hline $\begin{array}{l}\text { \# confirmed } \\
\text { symptomatic } \\
\text { hypoglycemia by gluco } \\
\text { check }\end{array}$ & 3 & 7 & 2 \\
\hline
\end{tabular}

$16 \%$ had no postprandial hyperglycemia ( $>120 \mathrm{mg} / \mathrm{dl}$ ) after iftar, $64 \%$ had 1 to 5 events, $12 \%$ had 6 to 10 events and $8 \%$ had more than 10 events. None needed admission for any reason.

\section{Conclusion:}

Many women with GDM fasted in Ramadan. However, hypoglycaemic events increased significantly during Ramadan fasting even for those on diet only or in those treated with metformin while assessed by free style Libre. The time between 12-18 hours had the highest incidence of hypoglycemic episodes. With the exception of lipids profile, there was no significant change in biochemical or biometric parameters. None was admitted to hospital.

This data are of important clinical implications and need to be repeated again with a larger cohort and with clear advice to re-check with SMBG if results indicate hypoglycaemia to confirm it and to assess the accuracy of free style Libre and or any other CGM in pregnant women with GDM during fasting Ramadan.

\section{References:}

1. Nor Azlin Mohamed Ismail et.al. Glycemic Control among Pregnant Diabetic Women on Insulin Who Fasted During Ramadan. Iran J Med Sci. 2011 Dec; 36(4): 254-259.

2. Sarita Bajaj et. Al. South Asian consensus statement on women's health and Ramadan. Indian J Endocrinol Metab. 2012 Jul-Aug; 16(4): 508-511. 